\title{
Extraction Methods and Inhibition Studies of Ten Plant Extracts on Nine Yam Rot Pathogenic Microorganisms
}

\author{
Dooshima Shiriki ${ }^{*}$, Simon Terver Ubwa ${ }^{2}$, Mohammed Ikagu Yusufu ${ }^{3}$, Tseaa Shambe ${ }^{2}$ \\ ${ }^{1}$ Department of Biological Sciences, Benue State University, Makurdi, Nigeria \\ ${ }^{2}$ Department of Chemistry, Benue State University, Makurdi, Nigeria \\ ${ }^{3}$ Department of Food Science and Technology, University of Agriculture, Makurdi, Nigeria \\ Email: *dshiriki@bsum.edu.ng
}

How to cite this paper: Shiriki, D., Ubwa, S.T., Yusufu, M.I. and Shambe, T. (2019) Extraction Methods and Inhibition Studies of Ten Plant Extracts on Nine Yam Rot Pathogenic Microorganisms. Food and Nutrition Sciences, 10, 439-458. https://doi.org/10.4236/fns.2019.104033

Received: November 8, 2018

Accepted: April 23, 2019

Published: April 26, 2019

Copyright $\odot 2019$ by author(s) and Scientific Research Publishing Inc. This work is licensed under the Creative Commons Attribution International License (CC BY 4.0).

http://creativecommons.org/licenses/by/4.0/

\begin{abstract}
Nine microorganisms, comprising of four bacteria, Erwinia carotovora, Pseudomonas aeruginosa, Serratia marcescens, Klebsiella oxytoca and five fungi, Rhizopus stolonifera, Aspergillus niger, Aspergillus flavus, Fusarium oxysporum and Penicillium marneffei, isolated from rotten yam tubers of 2016 harvest year, were treated with ten plants extracts (Passiflora edulis, Daniella oliveri, Ceiba pentandra, Jatropha tanjorensis, Azadrichta indica, Carica papaya, Moringa oleifera, Mangifera indica, Terminalia catapa and Senna alata), singly and synergistically by incorporation of extract in media for inhibition test. Two plant extracts singly and completely inhibited the growth of three organisms: Terminalia catapa at $100 \%$ and $10^{-1}$ showed complete inhibition (a) of Erwinia carotovora. Passiflora edulis at undiluted (100\%) concentration completely inhibited Rhizopus stolonifer and Penicillium marneffei, respectively. Synergistic plant extract recorded complete inhibition (a) of all the four bacteria isolates at $2 \mathrm{~mL}$ extract incorporation; ten (10) $\mathrm{mL}$ extract incorporation in media recorded complete inhibition (a) of three out of the five fungi isolates: Rhizopus stolonifer, Fusarium oxysporum and Penicillium marneffei, respectively; the other two fungi recorded high inhibition (b) of Aspergillus niger and Aspergillus flavus, respectively. Hot aqueous synergistic plants extract recorded poor inhibition of the isolates as compared to the cold. Soxhlet solvent extracted synergistic plants extract, however, recorded lower inhibition as compared to hot aqueous synergistic plants extract and cold aqueous synergistic plants extracts. Room temperature solvent extracted synergistic plants extracts recorded inhibition that was same as that obtained with cold aqueous synergistic plants extract. This research indicates that heat employed extractions recorded less inhibition activity.
\end{abstract}




\section{Keywords}

Microorganisms, Rotten Yam, Plant Extracts, Inhibition, Synergistic

\section{Introduction}

The use of plant extract for treatment of ailment is now preferred or recommended in fighting microbial infections instead of synthetic antimicrobial drugs that most microorganisms pathogenic to humans and perhaps even those pathogenic to plants are presently becoming resistant to [1]-[10]. There are about 250,000 to 500,000 species of plants on Earth [8] [9]. However, it is only a small percentage (1\% to $10 \%)$ of these plants that are used as foods by both humans and other animal species [9] [10]. The use of plant extracts, as well as other alternative forms of medical treatments, is enjoying great popularity in the late 1990s [9] [11]. The antimicrobial properties of plants have been investigated by researchers globally. In Latin America, a research tested 122 known plant species used for therapeutic treatments in Argentina [9] [12]. It was documented that among the compounds extracted from these plants, twelve inhibited the growth of Staphylococus aureus, ten inhibited Escherichia coli, and four inhibited Aspergillus niger, also was reported that the most potent compound was one extracted from Tabebuia impetiginosa. The antimicrobial properties of compounds obtained from Parthenum argentatum against Candida albicans, Torulopsis, Hansemula, Klebsiella pneumoniae and Pseudomonas aeruginosa were detected [9] [13] [14]. Research work conducted revealed that the substances extracted from nine known plants in Uruguai did not show any activity against $C$. albicans and Saccharomyces cerevisiae, but inhibited the growth of Bacillus subtilis, E. coli and $P$. aeruginosa [9] [14] [15] [16]. In many developing countries, traditional medicine is one of the primary healthcare systems [17] [18] [19]. Herbs are widely exploited in the traditional medicine and their curative potentials are well documented [20]. About $61 \%$ of new drugs developed between 1981 and 2002 were based on natural products and they had been very successful, especially in the areas of infectious disease and cancer [21]. Plants are rich in a wide variety of secondary metabolites such as tannins, terpenoids, alkaloids, flavonoids, glycosides, amongst others, which have been found in vitro to have antimicrobial properties [22] [23].

Therapeutic efficacy of many indigenous plants for several disorders has been described by practitioners of traditional medicine [24]. Antimicrobial properties of medicinal plants are being increasingly reported from different parts of the world. The World Health Organization estimates that plant extracts or their active constituents are used as folk medicine in traditional therapies of $80 \%$ of the world's population [25].

Most investigations in the use of plant extracts as alternative to synthetic drugs are bias for treatment of humans and animals with little attention to the 
treatment of plants and indeed food crops. Researchers who have worked on the use of some single plant extracts to inhibit yam rot microorganisms reported findings that yielded varying degrees of inhibition but no complete inhibition of all the rot causing microorganisms that exist in associative growth have been recorded [7] [26] [27] [28]. Hence, the use of some plants extracts reported to have antimicrobial activity for single and synergistic inhibition studies on the yam pathogenic microbes that were previously isolated from rotten yam tubers and confirmed as etiology of the rot in this study; also to probe further on for possible complete inhibition of the microorganisms that are present in associative growth; positive findings shall be recommended for adoption by farmers for fresh yam tuber preservation for long duration storage (shelf life elongation).

\section{Materials and Methods}

\subsection{Microorganisms}

Previously isolated and maintained yam rot pathogenic microorganisms: Nine microorganisms, comprising of four bacteria, Erwinia carotovora, Pseudomonas aeruginosa, Serratia marcescens, Klebsiella oxytoca and five fungi, Rhizopus stolonifera, Aspergillus niger, Aspergillus flavus, Fusarium oxysporum and Penicillium marneffei, of 2016 harvest year [29] were obtained from the laboratory, department of Biological Sciences, Benue State University, Makurdi where they were preserved and used for the antimicrobial inhibition test.

\subsection{Plant Extract Preparation}

\subsubsection{Single Plant Extract Preparation}

The method of [30] was used. Freshly harvested plant leaves of Passiflora edulis, Daniella oliveri, Ceiba pentandra, Jatropha tanjorensis, Azadrichta indica, Carica papaya, Moringa oleifera, Mangifera indica, Terminalia catapa and Senna alata were collected along $\mathrm{km} 4$ Gboko road Makurdi and were authenticated by a plant taxonomist in the Department of Biological Sciences, Benue State University, Makurdi and used. The leaves were washed in clean water and put in a clean plastic basket to drain. They were separately cut to fine shredding with a clean stainless steel knife and $10.0 \mathrm{~g}$ of each was weighed and wet milled in a warring blender with $50 \mathrm{~mL}$ sterile distilled water, the blends were individually filtered with No. 1 Whatman filter paper into labelled sterile $100 \mathrm{~mL}$ volumetric flask, the residue was washed with $30 \mathrm{~mL}$ of the sterile water, it was made up to mark using the sterile distilled water and labeled. Serial dilutions of $10^{-1}$ and $10^{-2}$ concentrations of the extracts were carried out by pipetting $1.0 \mathrm{~mL}$ of each filtrate into a $10 \mathrm{~mL}$ and $100 \mathrm{~mL}$ volumetric flask respectively and the volume was made up to mark with sterile distilled water. The extracts were stored up in the refrigerator and used for inhibition (antimicrobial activity) test.

\subsubsection{Cold Aqueous Synergistic Plants Extract Preparation}

The method of [31] was used with little modifications; plant leaves were blended in a ratio of 1:1. Fresh leaves of ten plants (Ceiba pentandra, Jatropha tanjoren- 
sis, Azadarichta indica, Moringa Oleifera, Carica papaya, Mangifera indica, Daniella oliveri, Terminalia catapa, Passiflora edulis and Senna alata) were collected and washed in clean sterile water, it was put to drain in a clean plastic basket for 10 minutes. The leaves were separately cut to fine shredding with a stainless steel kitchen knife and weighed (20.0 g each) individually using an electronic digital weighing balance. They were mix and blended using a warring blender into fine paste. The mix $(50.0 \mathrm{~g})$ was weighed into a sterilized $500 \mathrm{~mL}$ conical flask, 200 $\mathrm{mL}$ of sterile water was added and stirred for 10 minutes, it was plugged with cotton wool and kept to extract for 12 hours at room temperature. The extract was filtered with Whatman No 1 filter paper and the residue was washed with 50 $\mathrm{mL}$ of water to a concentration of $0.2 \mathrm{~g} / \mathrm{mL}$; the combined volume was decanted into a sterile bottle, properly corked; stored in the refrigerator and used for antimicrobial inhibition test.

\subsubsection{Hot Aqueous Synergistic Ten Plants Extraction}

The method of natives (Tiv, unpublished work), was used with little scientific modification (measurements and aseptic techniques). The blended mix (50.0 g) was weighed into a sterilized $500 \mathrm{~mL}$ beaker, $250 \mathrm{ml}$ of clean water was added, stirred and heated to boiling; it was allowed to boil on for 15 minutes for proper extraction. It was to cooled and filtered with Whatman No 1 filter paper into a $250 \mathrm{~mL}$ volumetric flask and made up to the mark with sterile water washings of the boiled plant mix. It was corked and stored in the refrigerator for antimicrobial inhibition test.

\subsubsection{Ethanol and Methanol Solvents Soxhlet Synergistic Ten Plants Extraction}

The method of [32] was used with little modification. The blended mix (100 g) were packed into timbles and placed inside the soxhlet extractor. The solvents (ethanol and methanol) were taken in round bottom flask of the extractor, respectively; heat was applied to the bottom of the flask from heating mantles and extraction was carried out for 10 hours. The solvents were recovered and the extracts were respectively turned into pre-weighed and labeled beakers, it was evaporated in evaporation bath for 24 hours. The extract was reconstituted with sterile water to a concentration of $0.2 \mathrm{~g} / \mathrm{ml}$ and used for the inhibition test.

\subsubsection{Room Temperature Ethanol and Methanol Solvents Synergistic Ten Plants Extraction}

The method of [33] was used with some modification. The blended mix (50.0 g) each was weighed into two sterilized $500 \mathrm{~mL}$ beaker respectively, $250 \mathrm{~mL}$ of solvents ethanol and methanol were added separately to individual beaker contents, stirred and left to extract on a shaker bath at room temperature for 12 hours. The extract was filtered through Whatman No. 1 filter paper into a sterilized conical flask, poured into pre weighed sterilized glass dishes respectively and exposed in laminar air flow chamber for the solvents evaporation. The yield was turned into a $250 \mathrm{~mL}$ volumetric flask and reconstituted with sterile water to a 
concentration of $0.2 \mathrm{~g} / \mathrm{mL}$ and stored away from light under room temperature to be used for the inhibition test.

\subsection{Plant Extract Incorporation}

\subsubsection{Single Plant Extract Incorporation}

The method of [27] was used. The plant extracts solution of $10 \mathrm{~g} / 50 \mathrm{~mL}$ of solvent (stock solution), and its serial dilutions of $10^{-1}$ and $10^{-2}$ were prepared by adding $1.0 \mathrm{~mL}$ each of the plant extract stock solutions in $10.0 \mathrm{~mL}$ and $100 \mathrm{~mL}$ standard volumetric flask then making it up to the mark with sterile distilled water respectively. A $1.0 \mathrm{~mL}$ of the undiluted stock and $1.0 \mathrm{~mL}$ each of the two dilutions of the single plant extracts were added into labelled sterile petri dishes respectively and molten nutrient Agar and potatoe dextrose agar held at $45^{\circ} \mathrm{C}$ was poured onto it, respectively; it was mixed properly by rotating the plates on the flat surface, the plates were allowed to set for 30 minutes, they were incubated for sterility check and only sterile plates were used for inhibition test.

\subsubsection{Synergistic Plant Extract Incorporation}

The method of [34] was used. The extract, $1.0 \mathrm{~mL}-10.0 \mathrm{~mL}$, each were pipette into sterile petri dishes and $20 \mathrm{~mL}$ or $15 \mathrm{~mL}$ (agar quantity reduced with increasing extract quantity) of molten nutrient agar or potato dextrose agar held at $45^{\circ} \mathrm{C}$ was poured onto it respectively and mixed thoroughly. The plates were allowed to set, packed and incubated overnight for sterility test and sterile plates only after incubation were used for the inhibition test.

\subsubsection{Inhibition Test}

The methods of [27] was used with little modifications; the bacteria and fungi isolates were seeded individually on the plants extract incorporated nutrient agar and potato dextrose agar respectively, controls were set up by seeding on media without plant extract addition; plates were incubated at $37^{\circ} \mathrm{C}$ for 48 hours for the bacteria and 7 days for the fungi. The plates were observed for microbial activity. Growth diameter where present was measured and it was compared with the control to device a percentage growth width using a transparent plastic ruler.

\section{Results and Discussion}

\subsection{Results}

\subsubsection{Single Plant Inhibition Test}

Table 1 present results of single plant extract inhibition (antimicrobial sensitivity) test of the ten plants (Passiflora edulis, Daniella oliveri, Ceiba pentandra, Jatropha tanjorencis, Azadarichta indica, Carica papaya, Moringa oleifera, Mangifera indica, Terminalia catapa and Senna alta) on the nine Isolates. Passiflora edulis at undiluted (100\%) concentration completely inhibited Rhizopus stolonifer and Penicillium marneffei; high inhibition was obtained in Rhizopus stolonifer and Penicillium marneffei at $10^{-1}$ dilution along with Aspergillus flavus and Fusarium oxysporum at undiluted concentrations. Good inhibition was recorded 
Table 1. Single plant extract inhibition test on the isolates.

\begin{tabular}{|c|c|c|c|c|c|c|}
\hline \multirow[t]{4}{*}{ Plant } & \multicolumn{6}{|c|}{ Organism/Extract concentration } \\
\hline & \multirow{2}{*}{\multicolumn{3}{|c|}{$\begin{array}{c}\text { Fungi } \\
\text { Rhizopus stolonifer }\end{array}$}} & \multirow{2}{*}{\multicolumn{3}{|c|}{$\begin{array}{c}\text { Bacteria } \\
\text { Erwinia carotovora }\end{array}$}} \\
\hline & & & & & & \\
\hline & $100 \%$ & $10^{-1}$ & $10^{-2}$ & $100 \%$ & $10^{-1}$ & $10^{-2}$ \\
\hline Passiflora edulis & $+++++^{\mathrm{a}}$ & $++++^{\mathrm{b}}$ & $+++^{c}$ & $+++^{c}$ & $++^{d}$ & $+^{\mathrm{e}}$ \\
\hline Daniella olivera & $-{ }^{f}$ & $-{ }^{f}$ & $-{ }^{f}$ & $--^{f}$ & $-f$ & $-{ }^{f}$ \\
\hline Ceiba pentandra & $--^{f}$ & $--^{f}$ & $--^{f}$ & $+^{\mathrm{e}}$ & $+^{\mathrm{e}}$ & $+^{\mathrm{e}}$ \\
\hline Jathropha tanjorensis & $+++^{c}$ & $+^{\mathrm{e}}$ & $--^{f}$ & $+++^{c}$ & $++^{d}$ & $++^{\mathrm{d}}$ \\
\hline Azadarichta indica & $+^{\mathrm{e}}$ & $--^{f}$ & $--^{f}$ & $+++^{c}$ & $+^{\mathrm{e}}$ & $-f$ \\
\hline Carica papaya & $+^{\mathrm{e}}$ & $-f$ & $-f$ & $++++^{\mathrm{b}}$ & $+^{\mathrm{e}}$ & $-\mathrm{f}$ \\
\hline Moringa oleifera & $-f$ & $-f$ & $-\mathrm{f}$ & $+++^{c}$ & $+^{\mathrm{e}}$ & $-\mathrm{f}$ \\
\hline Mangifera indica & $-f$ & $--^{f}$ & $-f$ & $+++^{c}$ & $+^{e}$ & $-{ }^{f}$ \\
\hline Terminalia catapa & $++^{\mathrm{d}}$ & $+^{\mathrm{e}}$ & $+^{\mathrm{e}}$ & $+++++^{\mathrm{a}}$ & $+++++^{\mathrm{a}}$ & $++++^{\mathrm{b}}$ \\
\hline \multirow[t]{2}{*}{ Senna alata } & $++++^{\mathrm{b}}$ & $+++^{c}$ & $++^{\mathrm{d}}$ & $+++^{c}$ & $+^{\mathrm{e}}$ & $+^{\mathrm{e}}$ \\
\hline & $a=1$ & $d=2$ & & $a=2$ & $d=3$ & \\
\hline \multirow[t]{3}{*}{ Summary } & $b=2$ & $e=5$ & & $b=2$ & $e=10$ & \\
\hline & $c=3$ & $\mathrm{f}=17$ & & $c=6$ & $f=7$ & \\
\hline & \multicolumn{3}{|c|}{ Aspergillus niger } & \multicolumn{3}{|c|}{ Pseudomonas aeruginosa } \\
\hline Passiflora edulis & $+++^{c}$ & $+++^{c}$ & $+^{\mathrm{e}}$ & $+^{\mathrm{e}}$ & $+^{\mathrm{e}}$ & $+^{\mathrm{e}}$ \\
\hline Daniella olivera & $+^{\mathrm{e}}$ & $-^{\mathrm{f}}$ & $-f$ & $++^{\mathrm{d}}$ & $++^{\mathrm{d}}$ & $+^{\mathrm{e}}$ \\
\hline Ceiba pentandra & $-f$ & $+^{\mathrm{e}}$ & $+^{\mathrm{e}}$ & $+++^{c}$ & $+++^{c}$ & $++^{\mathrm{d}}$ \\
\hline Jathropha tanjorensis & $-f$ & $-^{f}$ & $-f$ & $++++^{\mathrm{b}}$ & $++++^{\mathrm{b}}$ & $++++^{\mathrm{b}}$ \\
\hline Azadarichta indica & $+++^{c}$ & $++^{\mathrm{d}}$ & $-^{f}$ & $++^{\mathrm{d}}$ & $t^{\mathrm{e}}$ & $-\mathrm{f}$ \\
\hline Carica papaya & $+++^{c}$ & $++^{\mathrm{d}}$ & $-^{f}$ & $+++^{c}$ & $++^{d}$ & $+^{e}$ \\
\hline Moringa oleifera & $+++^{c}$ & $+^{\mathrm{e}}$ & $-f$ & $++^{\mathrm{d}}$ & $+^{\mathrm{e}}$ & $+^{\mathrm{e}}$ \\
\hline Mangifera indica & $++^{\mathrm{d}}$ & $+^{\mathrm{e}}$ & $-^{f}$ & $++^{\mathrm{d}}$ & $+^{\mathrm{e}}$ & $-^{\mathrm{f}}$ \\
\hline Terminalia catapa & $+^{\mathrm{e}}$ & $-^{\mathrm{f}}$ & $-f$ & $+++^{c}$ & $++^{d}$ & $+^{\mathrm{e}}$ \\
\hline \multirow[t]{2}{*}{ Senna alata } & $++++^{\mathrm{b}}$ & $++^{\mathrm{d}}$ & $+^{\mathrm{e}}$ & $++^{\mathrm{d}}$ & $t^{\mathrm{e}}$ & $t^{\mathrm{e}}$ \\
\hline & $a=0$ & $\mathrm{~d}=4$ & & $a=0$ & $d=9$ & \\
\hline \multirow[t]{3}{*}{ Summary } & $b=1$ & $e=8$ & & $b=3$ & $e=12$ & \\
\hline & $c=5$ & $f=12$ & & $c=4$ & $f=2$ & \\
\hline & \multicolumn{3}{|c|}{ Aspergillus flavus } & \multicolumn{3}{|c|}{ Serratia marcescens } \\
\hline Passiflora edulis & $++++^{b}$ & $+++^{c}$ & $+^{e}$ & $-^{f}$ & $-f$ & $-f$ \\
\hline Daniella olivera & $t^{\mathrm{e}}$ & $-^{\mathrm{f}}$ & $-^{\mathrm{f}}$ & $+^{\mathrm{e}}$ & $--^{\mathrm{f}}$ & $--^{\mathrm{f}}$ \\
\hline Ceiba pentandra & $+^{e}$ & $+^{\mathrm{e}}$ & $+^{\mathrm{e}}$ & $++^{\mathrm{d}}$ & $+^{\mathrm{e}}$ & $-f$ \\
\hline Jathropha tanjorensis & $++^{\mathrm{d}}$ & $+^{\mathrm{e}}$ & $+^{\mathrm{e}}$ & $+^{\mathrm{e}}$ & $-f$ & $-f$ \\
\hline Azadarichta indica & $+++^{c}$ & $++^{\mathrm{d}}$ & $-^{\mathrm{f}}$ & $+^{\mathrm{e}}$ & $+^{\mathrm{e}}$ & $-^{\mathrm{f}}$ \\
\hline Carica papaya & $+++^{c}$ & $++^{\mathrm{d}}$ & $-^{f}$ & $++^{\mathrm{d}}$ & $+^{e}$ & $-^{f}$ \\
\hline Moringa oleifera & $+++^{c}$ & $++^{d}$ & $-^{\mathrm{f}}$ & $+^{\mathrm{e}}$ & $+^{\mathrm{e}}$ & $-^{\mathrm{f}}$ \\
\hline Mangifera indica & $++^{\mathrm{d}}$ & $+^{\mathrm{e}}$ & $-^{\mathrm{f}}$ & $+^{\mathrm{e}}$ & $+^{\mathrm{e}}$ & $+^{\mathrm{e}}$ \\
\hline Terminalia catapa & $++^{\mathrm{d}}$ & $+^{e}$ & $-^{\mathrm{f}}$ & $-^{\mathrm{f}}$ & $-^{\mathrm{f}}$ & $-^{f}$ \\
\hline \multirow[t]{2}{*}{ Senna alata } & $+++^{c}$ & $++^{\mathrm{d}}$ & $+^{\mathrm{e}}$ & $++^{\mathrm{d}}$ & $+^{\mathrm{e}}$ & $+^{\mathrm{e}}$ \\
\hline & $a=0$ & $\mathrm{~d}=7$ & & $a=0$ & $d=3$ & \\
\hline \multirow[t]{2}{*}{ Summary } & $b=1$ & $e=10$ & & $b=0$ & $e=13$ & \\
\hline & $c=5$ & $\mathrm{f}=7$ & & $c=0$ & $f=14$ & \\
\hline
\end{tabular}




\begin{tabular}{|c|c|c|c|c|c|c|}
\hline \multirow[b]{2}{*}{ Passiflora edulis } & \multicolumn{3}{|c|}{ Fusarium oxysporum } & \multicolumn{3}{|c|}{ Klebsiella oxytoca } \\
\hline & $++++^{\mathrm{b}}$ & $++^{\mathrm{d}}$ & $++^{d}$ & $+^{\mathrm{e}}$ & $+^{\mathrm{e}}$ & $+^{\mathrm{e}}$ \\
\hline Daniella olivera & $+^{\mathrm{e}}$ & $+^{\mathrm{e}}$ & $++^{\mathrm{d}}$ & $+^{e}$ & $+^{\mathrm{e}}$ & $+^{\mathrm{e}}$ \\
\hline Ceiba pentandra & $+++^{c}$ & $+++^{c}$ & $+^{\mathrm{e}}$ & $++^{d}$ & $+^{e}$ & $+^{\mathrm{e}}$ \\
\hline Jathropha tanjorensis & $+++^{c}$ & $++^{\mathrm{d}}$ & $+^{e}$ & $++++^{\mathrm{b}}$ & $++^{\mathrm{d}}$ & $++^{\mathrm{d}}$ \\
\hline Azadarichta indica & $++^{\mathrm{d}}$ & $+^{\mathrm{e}}$ & $+^{\mathrm{e}}$ & $++^{\mathrm{d}}$ & $+^{\mathrm{e}}$ & $-{ }^{f}$ \\
\hline Carica papaya & $++^{\mathrm{d}}$ & $++^{\mathrm{d}}$ & $t^{\mathrm{e}}$ & $+++^{c}$ & $++^{d}$ & $+^{\mathrm{e}}$ \\
\hline Moringa oleifera & $+^{\mathrm{e}}$ & $+^{\mathrm{e}}$ & $-{ }^{f}$ & $+^{\mathrm{e}}$ & $+^{e}$ & $-f$ \\
\hline Mangifera indica & $+^{e}$ & $+^{\mathrm{e}}$ & $-f$ & $+^{e}$ & $t^{e}$ & $+^{e}$ \\
\hline Terminalia catapa & $+^{\mathrm{e}}$ & $+^{\mathrm{e}}$ & $t^{e}$ & $+^{\mathrm{e}}$ & $+^{e}$ & $+^{\mathrm{e}}$ \\
\hline Senna alata & $+++^{c}$ & $++^{\mathrm{d}}$ & $+^{\mathrm{e}}$ & $+^{\mathrm{e}}$ & $+^{\mathrm{e}}$ & $t^{e}$ \\
\hline \multirow{3}{*}{ Summary } & $a=0$ & $\mathrm{~d}=8$ & & $a=0$ & $d=5$ & \\
\hline & $b=1$ & $e=15$ & & $b=1$ & $\mathrm{e}=21$ & \\
\hline & $c=4$ & $f=2$ & & $c=1$ & $f=2$ & \\
\hline \multicolumn{7}{|c|}{ Penicillium marneffei } \\
\hline Passiflora edulis & $+++++^{a}$ & $++++^{b}$ & $+++^{c}$ & & & \\
\hline Daniella olivera & $-f$ & $-f$ & $-f$ & & & \\
\hline Ceiba pentandra & $-f$ & $-^{\mathrm{f}}$ & $-f$ & & & \\
\hline Jathropha tanjorensis & $++++^{b}$ & $+++^{c}$ & $+^{e}$ & & & \\
\hline Azadarichta indica & $+^{e}$ & $+^{\mathrm{e}}$ & $-f$ & & & \\
\hline Carica papaya & $+^{\mathrm{e}}$ & $--^{f}$ & $-^{\mathrm{f}}$ & & & \\
\hline Moringa oleifera & $--^{f}$ & $-f$ & $-f$ & & & \\
\hline Mangifera indica & $--^{\mathrm{f}}$ & $--^{f}$ & $-^{\mathrm{f}}$ & & & \\
\hline Terminalia catapa & $+++^{c}$ & $++^{\mathrm{d}}$ & $+^{\mathrm{e}}$ & & & \\
\hline \multirow[t]{2}{*}{ Senna alata } & $+^{\mathrm{e}}$ & $-f$ & $-f$ & & & \\
\hline & $a=1$ & $d=1$ & & & & \\
\hline \multirow[t]{2}{*}{ Summary } & $\mathrm{b}=2$ & $e=6$ & & & & \\
\hline & $c=3$ & $f=17$ & & & & \\
\hline
\end{tabular}

Summary of overall inhibition: $\mathrm{a}=4, \mathrm{~b}=13, \mathrm{c}=31, \mathrm{~d}=41, \mathrm{e}=100, \mathrm{f}=60$; Key: = no inhibition; $+=$ mild inhibition $(0.5 \%-34 \%$ inhibition $)$; $++=$ moderate inhibition $(35 \%-54 \%$ inhibition $)$; $+++=$ good inhibition (55\% - 74\% inhibition); $++++=$ high inhibition (75\% - 99\% inhibition); $+++++=$ complete inhibition; $\mathrm{NA}=$ not applicable; $\mathrm{a}=$ complete inhibition, $\mathrm{b}=$ high inhibition, $\mathrm{c}=$ good inhibition, $\mathrm{d}=$ moderate inhibition; $\mathrm{e}=$ mild inhibition, $\mathrm{f}=$ no inhibition.

by Rhizopus stolonifer at $10^{-2}$ dilution, Aspergillus niger at undiluted and $10^{-1}$ concentrations along with Aspergillus flavus, and Penicillium marneffei and the bacteria Erwinia carotovora at $10^{-2}$ and undiluted concentrations.

Daniella olivera recorded mild to moderate inhibition of the organisms with the exception of Rhizopus stolonifer, Erwinia carotovora and Penicillium marneffei that recorded no inhibition respectively.

Ceiba pentandra showed good inhibition (c) of Pseudomonas aeruginosa and Fusarium oxysporum at $100 \%$ and $10^{-1}$ dilutions, respectively. Other organisms recorded mild (e) to moderate inhibition (d), respectively; with the exception of Penicillium marneffei that recorded no inhibition (f). 
Jatropha tanjorensis at undiluted concentration (100\%) showed high inhibition (b) of Pseudomonas aeruginosa (both at 100\% and the dilutions), along with Klebsiella oxytoca and Penicillium marneffei. It also recorded good inhibition (c) at $100 \%$ concentration on Rhizopus stolonifer, Erwinia carotovora, Fusarium oxysporum and Penicillium marneffei at $10^{-1}$ dilution, respectively.

Azadarichta indica in the undiluted concentration only, showed good inhibition (c) of Erwinia carotovora, Aspergillus niger and Aspergillus flavus, respectively.

Carica papaya at undiluted concentration recorded high inhibition (b) of Erwinia carotovora, and good inhibition (c) of Aspergillus niger, Pseudomonas aeruginosa and Klebsiella oxytoca, respectively.

Moringa oleifera at undiluted concentration only recorded good inhibition (c) of Erwinia carotovora and Aspergillus niger. Other organisms recorded moderate to mild inhibition with the exceptions of Rhizopus stolonifer and Penicillium marneffei; that showed no inhibition (resistant).

Mangifera indica at undiluted concentration recorded good inhibition (c) of Erwinia carotovora, moderate to mild inhibition of the other organisms with the exception of Penicillium marneffei that showed no inhibition.

Terminalia catapa at $100 \%$ and $10^{-1}$ showed complete inhibition (a) of Erwinia carotovora, and recorded high inhibition (b) at $10^{-2}$ dilution. It recorded good inhibition (c) of Pseudomonas aeruginosa and Penicillium marneffei, respectively. It also recorded mild to moderate inhibition of the other organisms with the exception of Serratia marcescens which recorded no inhibition (resistant).

Senna alata at undiluted concentration recorded high inhibition (b) of Rhizopus stolonifer and Aspergillus niger. It showed good inhibition (c) of Rhizopus stolonifer at $10^{-1}$ dilution and Erwinia carotovora, Aspergillus flavus and Fusarium oxysporum at $100 \%$ concentrations, respectively. It recorded mild to moderate inhibition of other organisms.

\subsubsection{Cold Aqueous Synergistic Ten Plants Inhibition Test on Isolates}

Table 2 present results of cold aqueous synergistic ten plants extract inhibition test on the nine isolated microorganism.

Synergistic effect of the ten plants extract on the isolates reveals that $1.0 \mathrm{~mL}$ addition of extracts in the culture media recorded moderate inhibition (d) of Erwinia carotovora and Klebsiella oxytoca, and mild inhibition (e) of Pseudomonas aeruginosa and Serratia marcescens, respectively; the fungi isolates however showed no inhibition (f). $2.0 \mathrm{~mL}$ incorporation of the extract recorded complete inhibition (a) of all the four bacteria isolates (Erwinia carotovora, Pseudomonas aeruginosa, Serratia marcescens and Klebsiella oxytoca), the fungi isolates showed no inhibition (f). Addition of $3.0 \mathrm{~mL}$ of extract showed mild inhibition (e) of Aspergillus niger and Penicillium marneffei without affecting the other three fungi; $4.0 \mathrm{~mL}$ incorporation of the extract showed moderate inhibition (d) of Aspergillius niger, Aspergillus flavus and Penicillium marneffei and mild inhibition (e) of Rhizopus stolonifer and Fusarium oxytoca, respectively. 
Table 2. Cold aqueous synergistic ten plants extract inhibition test on isolates.

\begin{tabular}{|c|c|c|c|c|c|c|c|c|c|c|}
\hline \multirow{2}{*}{ Microorganism } & \multicolumn{10}{|c|}{ Extract volume applied } \\
\hline & $1 \mathrm{~mL}$ & $2 \mathrm{~mL}$ & $3 \mathrm{~mL}$ & $4 \mathrm{~mL}$ & $5 \mathrm{~mL}$ & $6 \mathrm{~mL}$ & $7 \mathrm{~mL}$ & $8 \mathrm{~mL}$ & $9 \mathrm{~mL}$ & $10 \mathrm{~mL}$ \\
\hline Rhizopus stolonifer & $-^{f}$ & $-^{f}$ & $-^{f}$ & $+^{\mathrm{e}}$ & $+++^{c}$ & $+++^{c}$ & $+++^{c}$ & $+++^{c}$ & $++++^{\mathrm{b}}$ & $+++++^{\mathrm{a}}$ \\
\hline Aspergillus niger & $-{ }^{f}$ & $-f^{f}$ & $t^{e}$ & $+^{e}$ & $+++^{c}$ & $+++^{c}$ & $++++^{\mathrm{b}}$ & $++++^{\mathrm{b}}$ & $++++^{\mathrm{b}}$ & $++++^{\mathrm{b}}$ \\
\hline Aspergillus flavus & $-^{f}$ & $--^{f}$ & $-^{f}$ & $+^{e}$ & $+++^{c}$ & $++++^{\mathrm{b}}$ & $++++^{\mathrm{b}}$ & $++++^{\mathrm{b}}$ & $++++^{\mathrm{b}}$ & $++++^{\mathrm{b}}$ \\
\hline Fusarium oxysporum & $-^{f}$ & $-{ }^{f}$ & $--^{f}$ & $+^{\mathrm{e}}$ & $+++^{c}$ & $+++^{c}$ & $++++^{\mathrm{b}}$ & $++++^{\mathrm{b}}$ & $+++++^{\mathrm{a}}$ & $+++++^{\mathrm{a}}$ \\
\hline Penicillium marneffei & $-^{f}$ & $-{ }^{f}$ & $+^{e}$ & $++^{\mathrm{d}}$ & $++++^{\mathrm{b}}$ & $++++^{\mathrm{b}}$ & $++++^{\mathrm{b}}$ & $++++^{\mathrm{b}}$ & $+++++^{\mathrm{a}}$ & $+++++^{\mathrm{a}}$ \\
\hline Erwinia carotovora & $+^{\mathrm{d}}$ & $+++++^{\mathrm{a}}$ & $N A$ & $N A$ & $N A$ & $N A$ & $N A$ & $N A$ & $N A$ & $N A$ \\
\hline Pseudomonas aeruginosa & $--^{f}$ & $+++++^{\mathrm{a}}$ & $N A$ & $N A$ & $N A$ & $N A$ & $N A$ & $N A$ & $N A$ & $N A$ \\
\hline Serratia marcescens & $-^{f}$ & $+++++^{\mathrm{a}}$ & $N A$ & $N A$ & $N A$ & $N A$ & $N A$ & $N A$ & $N A$ & $N A$ \\
\hline Klebsiella oxytoca & $+^{\mathrm{d}}$ & $+++++^{\mathrm{a}}$ & $N A$ & $N A$ & $N A$ & $N A$ & $N A$ & $N A$ & $N A$ & $N A$ \\
\hline
\end{tabular}

Summary of inhibition: $\mathrm{a}=9, \mathrm{~b}=15, \mathrm{c}=9, \mathrm{~d}=5, \mathrm{e}=6, \mathrm{f}=13$; Key: $=$ no inhibition; $+=$ mild inhibition $(0.5 \%-34 \%$ inhibition $) ;++=$ moderate inhibition (35\% - 54\% inhibition); $+++=$ good inhibition (55\% - 74\% inhibition); ++++ = high inhibition (75\% - 99\% inhibition); +++++ = complete inhibition; NA = not applicable; $\mathrm{a}=$ complete inhibition, $\mathrm{b}=$ high inhibition, $\mathrm{c}=$ good inhibition, $\mathrm{d}=$ moderate inhibition; $\mathrm{e}=$ mild inhibition, $\mathrm{f}=$ no inhibition.

There was marked increase in the inhibition with the addition of $5.0 \mathrm{~mL}$ of the extract, showing high inhibition (b) of Penicillium marneffei and good inhibition (c) of Rhizopus stolonifer, Aspergillus niger, Aspergilus flavus and Fusarium oxysporum, respectively. Incorporation of $6 \mathrm{~mL}$ of the extract showed high inhibition (b) of Aspergillus flavus and Penicillium marneffei, it recorded good inhibition (c) of Rhizopus stolonifer, Aspergillus niger and Fusarium oxysporum, respectively. Addition of $7 \mathrm{~mL}$ and $8 \mathrm{~mL}$ of extract to test media showed high inhibition (b) of Aspergillius niger, Aspergillus flavus, Fusarium oxysporum and Penicillium marneffei, it recorded good inhibition (c) of Rhizopus stolonifer, respectively.

Incorporation of $9 \mathrm{~mL}$ recorded complete inhibition (a) of Fusarium oxysporum and Penicillium marneffei and high inhibition (b) of Rhizopus stolonifer, Aspergillus niger and Aspergilus flavus, respectively. Incorporation of $10 \mathrm{~mL}$ of extract recorded complete inhibition (a) of Rhizopus stolonifer, Fusarium oxysporum and Penicillium marneffei and high inhibition (b) of Aspergillus niger and Aspergilus flavus, respectively.

\subsubsection{Hot Aqueous Synergistic Ten Plants Extract Test}

Table 3 shows results of hot aqueous synergistic ten plant extract inhibition test on the isolates. There was no inhibition of any of the isolated organism by $1 \mathrm{~mL}$ incorporation; addition of $2 \mathrm{~mL}$ of extract showed mild inhibition (e) of the four bacteria isolates (Erwinia carotovora Pseudomonas aeruginosa, Serratia marcescens and Klebsiella oxytoca,) respectively, there was no inhibition of the fungi. Addition of $3.0 \mathrm{~mL}$ of extract showed complete inhibition (a) of the four bacteria isolates, the fungi isolates recorded no inhibition. Incorporation of $4 \mathrm{~mL}$ recorded mild inhibition (e) of Penicillium marneffei, the other four fungi showed no inhibition. Addition of $5 \mathrm{~mL}$ extract recorded moderate inhibition of 
Table 3. Hot aqueous synergistic Ten-Plant extract inhibition test.

\begin{tabular}{|c|c|c|c|c|c|c|c|c|c|c|}
\hline \multirow{2}{*}{ Microorganism } & \multicolumn{10}{|c|}{ Extract volume applied } \\
\hline & $1 \mathrm{~mL}$ & $2 \mathrm{~mL}$ & $3 \mathrm{~mL}$ & $4 \mathrm{~mL}$ & $5 \mathrm{~mL}$ & $6 \mathrm{~mL}$ & $7 \mathrm{~mL}$ & $8 \mathrm{~mL}$ & $9 \mathrm{~mL}$ & $10 \mathrm{~mL}$ \\
\hline Rhizopus stolonifer & $-{ }^{f}$ & $-{ }^{\mathrm{f}}$ & $-^{\mathrm{f}}$ & $-{ }^{\mathrm{f}}$ & $+^{e}$ & $+++^{\mathrm{c}}$ & $+++^{\mathrm{c}}$ & $++++^{\mathrm{b}}$ & $++++^{\mathrm{b}}$ & $++++^{\mathrm{b}}$ \\
\hline Aspergillus niger & $-{ }^{\mathrm{f}}$ & $-{ }^{\mathrm{f}}$ & $-{ }^{\mathrm{f}}$ & $-{ }^{\mathrm{f}}$ & $+^{\mathrm{e}}$ & $+++^{\mathrm{c}}$ & $+++^{\mathrm{c}}$ & $+++^{\mathrm{c}}$ & $++++^{\mathrm{b}}$ & $++++^{\mathrm{b}}$ \\
\hline Aspergillus flavus & $-{ }^{\mathrm{f}}$ & $-{ }^{\mathrm{f}}$ & $-{ }^{\mathrm{f}}$ & $-{ }^{\mathrm{f}}$ & $+^{e}$ & $+++^{\mathrm{c}}$ & $+++^{\mathrm{c}}$ & $++++^{\mathrm{b}}$ & $++++^{\mathrm{b}}$ & $++++^{\mathrm{b}}$ \\
\hline Fusarium oxysporum & $--^{\mathrm{f}}$ & $--^{f}$ & $--^{f}$ & $-{ }^{\mathrm{f}}$ & $++^{\mathrm{d}}$ & $+++^{c}$ & $+++^{c}$ & $++++^{\mathrm{b}}$ & $++++^{\mathrm{b}}$ & $+++++^{\mathrm{a}}$ \\
\hline Penicillium marneffei & $-{ }^{\mathrm{f}}$ & $--^{f}$ & $--^{f}$ & $+^{\mathrm{e}}$ & $++^{\mathrm{d}}$ & $+++^{c}$ & $++++^{\mathrm{b}}$ & $++++^{\mathrm{b}}$ & $++++^{\mathrm{b}}$ & $+++++^{\mathrm{a}}$ \\
\hline Erwinia carotovora & $-{ }^{f}$ & $+^{e}$ & $+++++^{\mathrm{a}}$ & $N A$ & $N A$ & $N A$ & $N A$ & $N A$ & $N A$ & $N A$ \\
\hline $\begin{array}{c}\text { Pseudomonas } \\
\text { aeruginosa }\end{array}$ & $-^{\mathrm{f}}$ & $+^{\mathrm{e}}$ & $+++++^{\mathrm{a}}$ & $N A$ & $N A$ & $N A$ & $N A$ & $N A$ & $N A$ & $N A$ \\
\hline Serratia marcescens & $--^{f}$ & $+^{e}$ & $+++++^{\mathrm{a}}$ & $N A$ & $N A$ & $N A$ & $N A$ & $N A$ & $N A$ & $N A$ \\
\hline Klebsiella oxytoca & $-f$ & $+^{e}$ & $+++++^{\mathrm{a}}$ & $N A$ & $N A$ & $N A$ & $N A$ & $N A$ & $N A$ & $N A$ \\
\hline
\end{tabular}

Summary of inhibition: $\mathrm{a}=6, \mathrm{~b}=13, \mathrm{c}=10, \mathrm{~d}=2, \mathrm{e}=8, \mathrm{f}=23$; Key: = no inhibition; $+=$ mild inhibition $(0.5 \%-34 \%$ inhibition $)$; $++=$ moderate inhibition (35\% - 54\% inhibition); +++ = good inhibition (55\% - 74\% inhibition); ++++ = high inhibition $(75 \%-99 \%$ inhibition); $+++++=$ complete inhibition; NA = not applicable; $\mathrm{a}=$ complete inhibition, $\mathrm{b}=$ high inhibition, $\mathrm{c}=$ good inhibition, $\mathrm{d}=$ moderate inhibition; $\mathrm{e}=$ mild inhibition, $\mathrm{f}=$ no inhibition.

Fusarium oxysporum and Penicillium marneffei, with mild inhibition (e) of Fusarium oxytoca, Aspergillius niger and Aspergillus flavus, respectively. Incorporation of $6 \mathrm{~mL}$ and $7 \mathrm{~mL}$ recorded good inhibition (c) of the fungi isolates with the exception of Penicillium marneffei which recorded high inhibition (b) at 7 $\mathrm{mL}$ extract incorporation.

Incorporation of $8 \mathrm{~mL}$ and $9 \mathrm{~mL}$ extract, recorded high inhibition (b) of the fungi isolates with the exception of Aspergillus niger which recorded good inhibition (c) with $8 \mathrm{~mL}$ extract addition. Addition of $10 \mathrm{~mL}$ of extract recorded complete inhibition of Fusarium oxysporum and Penicillium marneffe; it also recorded high inhibition (b) of Rhizopus stolonifer, Aspergillius niger and Aspergillus flavus, respectively.

\subsubsection{Ethanol and Methanol Solvents Soxhlet Extracted Synergistic Ten-Plants Inhibition Test on the Isolates}

Table 4 and Table 5 present results of ethanol and methanol solvents - soxhlet extracted synergistic ten plants inhibition test on the isolates; results obtained from both solvents were the same. Incorporation of $1 \mathrm{~mL}$ extract showed no inhibition for all isolates. Addition of $2 \mathrm{~mL}$ extract recorded mild inhibition (e) of Erwinia carotovora and Klebsiella oxytoca only. Incorporation of $3 \mathrm{~mL}$ recorded good inhibition (c) of Klebsiella oxytoca; moderate inhibition (d) of Erwinia carotovora and mild inhibition (e) of Pseudomonas aeruginosa, Serratia marcescens the fungi recorded no inhibition. Incorporation of $4 \mathrm{~mL}$ of extract recorded high inhibition (b) of Erwinia carotovora and Klebsiella oxytoca, it showed good inhibition (c) of Pseudomonas aeruginosa and Serratia marcescens; and mild inhibition (e) of the fungi Penicillium marneffei, respectively. Addition of $5 \mathrm{~mL}$ extract completely inhibited the four bacteria isolates; it recorded moderate inhibition of Penicillium marneffei and mild inhibition of Rhizopus stolonifer, 
Table 4. Ethanol soxhlet extracted synergistic Ten-Plants inhibition test on the isolates.

\begin{tabular}{|c|c|c|c|c|c|c|c|c|c|c|}
\hline \multirow{2}{*}{ Microorganism } & \multicolumn{10}{|c|}{ Extract volume applied } \\
\hline & $1 \mathrm{~mL}$ & $2 \mathrm{~mL}$ & $3 \mathrm{~mL}$ & $4 \mathrm{~mL}$ & $5 \mathrm{~mL}$ & $6 \mathrm{~mL}$ & $7 \mathrm{~mL}$ & $8 \mathrm{~mL}$ & $9 \mathrm{~mL}$ & $10 \mathrm{~mL}$ \\
\hline Rhizopus stolonifer & $-^{\mathrm{f}}$ & $-^{\mathrm{f}}$ & $-{ }^{\mathrm{f}}$ & $-^{\mathrm{f}}$ & $t^{e}$ & $+++^{\mathrm{c}}$ & $+++^{\mathrm{c}}$ & $+++^{c}$ & $++++^{\mathrm{b}}$ & $++++^{\mathrm{b}}$ \\
\hline Aspergillus niger & $-{ }^{\mathrm{f}}$ & $-{ }^{\mathrm{f}}$ & $-{ }^{\mathrm{f}}$ & $-{ }^{\mathrm{f}}$ & $+^{\mathrm{e}}$ & $+^{\mathrm{e}}$ & $++^{\mathrm{d}}$ & $++++^{\mathrm{b}}$ & $++++^{\mathrm{b}}$ & $++++^{\mathrm{b}}$ \\
\hline Aspergillus flavus & $-{ }^{\mathrm{f}}$ & $-{ }^{\mathrm{f}}$ & $-{ }^{\mathrm{f}}$ & $--^{f}$ & $+^{e}$ & $t^{e}$ & $++^{\mathrm{d}}$ & $+++^{c}$ & $++++^{\mathrm{b}}$ & $++++^{\mathrm{b}}$ \\
\hline Fusarium oxysporum & $--^{\mathrm{f}}$ & $--^{\mathrm{f}}$ & $--^{\mathrm{f}}$ & $--^{\mathrm{f}}$ & $+^{\mathrm{e}}$ & $++^{\mathrm{d}}$ & $+++^{\mathrm{c}}$ & $+++^{c}$ & $++++^{\mathrm{b}}$ & $+++++^{\mathrm{a}}$ \\
\hline Penicillium marneffei & $--^{f}$ & $-{ }^{\mathrm{f}}$ & $--^{f}$ & $+^{\mathrm{e}}$ & $++^{\mathrm{d}}$ & $++^{\mathrm{d}}$ & $+++^{\mathrm{c}}$ & $+++^{c}$ & $++++^{\mathrm{b}}$ & $++++^{\mathrm{b}}$ \\
\hline Erwinia carotovora & $-{ }^{\mathrm{f}}$ & $+^{e}$ & $++^{\mathrm{d}}$ & $++++^{\mathrm{b}}$ & $+++++^{\mathrm{a}}$ & $N A$ & $N A$ & $N A$ & $N A$ & $N A$ \\
\hline $\begin{array}{c}\text { Pseudomonas } \\
\text { aeruginosa }\end{array}$ & $-{ }^{\mathrm{f}}$ & $-f^{\mathrm{f}}$ & $+^{\mathrm{e}}$ & $+++^{c}$ & $+++++^{\mathrm{a}}$ & $N A$ & $N A$ & $N A$ & $N A$ & $N A$ \\
\hline Serratia marcescens & $--^{\mathrm{f}}$ & $-^{\mathrm{f}}$ & $+^{e}$ & $+++^{c}$ & $+++++^{\mathrm{a}}$ & $N A$ & $N A$ & $N A$ & $N A$ & $N A$ \\
\hline Klebsiella oxytoca & $-{ }^{f}$ & $+^{e}$ & $+++^{c}$ & $++++^{\mathrm{b}}$ & $+++++^{\mathrm{a}}$ & $N A$ & $N A$ & $N A$ & $N A$ & $N A$ \\
\hline
\end{tabular}

Summary of inhibition: $\mathrm{a}=5, \mathrm{~b}=12, \mathrm{c}=11, \mathrm{~d}=4, \mathrm{e}=11, \mathrm{f}=25$; Key: $=$ no inhibition; $+=$ mild inhibition $(0.5 \%-34 \%$ inhibition $)$; $++=$ moderate inhibition (35\% - 54\% inhibition); +++ = good inhibition (55\% - 74\% inhibition); ++++ = high inhibition (75\% - 99\% inhibition); +++++ = complete inhibition; $\mathrm{NA}=$ not applicable; $\mathrm{a}=$ complete inhibition; $\mathrm{b}=$ high inhibition; $\mathrm{c}=$ good inhibition; $\mathrm{d}=$ moderate inhibition; $\mathrm{e}=$ mild inhibition; $\mathrm{f}=$ no inhibition .

Table 5. Methanol soxhlet extracted synergistic Ten-Plant inhibition test on the isolates.

\begin{tabular}{|c|c|c|c|c|c|c|c|c|c|c|}
\hline \multirow{2}{*}{ Microorganism } & \multicolumn{10}{|c|}{ Extract volume applied } \\
\hline & $1 \mathrm{~mL}$ & $2 \mathrm{~mL}$ & $3 \mathrm{~mL}$ & $4 \mathrm{~mL}$ & $5 \mathrm{~mL}$ & $6 \mathrm{~mL}$ & $7 \mathrm{~mL}$ & $8 \mathrm{~mL}$ & $9 \mathrm{~mL}$ & $10 \mathrm{~mL}$ \\
\hline Rhizopus stolonifer & $-{ }^{\mathrm{f}}$ & $-{ }^{\mathrm{f}}$ & $-{ }^{\mathrm{f}}$ & $--^{\mathrm{f}}$ & $+^{\mathrm{e}}$ & $++^{\mathrm{d}}$ & $+++^{c}$ & $+++^{\mathrm{c}}$ & $++++^{\mathrm{b}}$ & $++++^{\mathrm{b}}$ \\
\hline Aspergillus niger & $-{ }^{f}$ & $--^{\mathrm{f}}$ & $--^{f}$ & $--^{f}$ & $+^{e}$ & $+^{e}$ & $++^{\mathrm{d}}$ & $++++^{\mathrm{b}}$ & $++++^{\mathrm{b}}$ & $++++^{\mathrm{b}}$ \\
\hline Aspergillus flavus & $-{ }^{f}$ & $-{ }^{\mathrm{f}}$ & $-{ }^{\mathrm{f}}$ & $-{ }^{\mathrm{f}}$ & $+^{e}$ & $+^{e}$ & $++^{\mathrm{d}}$ & $+++^{\mathrm{c}}$ & $++++^{\mathrm{b}}$ & $++++^{\mathrm{b}}$ \\
\hline Fusarium oxysporum & $-{ }^{\mathrm{f}}$ & $-{ }^{\mathrm{f}}$ & $-{ }^{\mathrm{f}}$ & $-{ }^{\mathrm{f}}$ & $+^{e}$ & $++^{\mathrm{d}}$ & $+++^{\mathrm{c}}$ & $+++^{c}$ & $++++^{\mathrm{b}}$ & $+++++^{\mathrm{a}}$ \\
\hline Penicillium marneffei & $-{ }^{\mathrm{f}}$ & $-{ }^{\mathrm{f}}$ & $-{ }^{\mathrm{f}}$ & $+^{\mathrm{e}}$ & $++^{\mathrm{d}}$ & $++^{\mathrm{d}}$ & $+++^{c}$ & $+++^{\mathrm{c}}$ & $++++^{\mathrm{b}}$ & $++++^{\mathrm{b}}$ \\
\hline Erwinia carotovora & $-{ }^{\mathrm{f}}$ & $+^{\mathrm{e}}$ & $++^{\mathrm{d}}$ & $++++^{\mathrm{b}}$ & $+++++^{\mathrm{a}}$ & $N A$ & $N A$ & $N A$ & $N A$ & $N A$ \\
\hline $\begin{array}{c}\text { Pseudomonas } \\
\text { aeruginosa }\end{array}$ & $-{ }^{\mathrm{f}}$ & $-{ }^{\mathrm{f}}$ & $+^{\mathrm{e}}$ & $+++^{c}$ & $+++++^{\mathrm{a}}$ & $N A$ & $N A$ & $N A$ & $N A$ & $N A$ \\
\hline Serratia marcescens & $-{ }^{\mathrm{f}}$ & $-{ }^{\mathrm{f}}$ & $+^{\mathrm{e}}$ & $+++^{c}$ & $+++++^{\mathrm{a}}$ & $N A$ & $N A$ & $N A$ & $N A$ & $N A$ \\
\hline Klebsiella oxytoca & $-{ }^{\mathrm{f}}$ & $+^{e}$ & $+++^{\mathrm{c}}$ & $++++^{\mathrm{b}}$ & $+++++^{\mathrm{a}}$ & $N A$ & $N A$ & $N A$ & $N A$ & $N A$ \\
\hline
\end{tabular}

Summary of inhibition: $\mathrm{a}=5, \mathrm{~b}=12, \mathrm{c}=10, \mathrm{~d}=7, \mathrm{e}=10, \mathrm{f}=25 \mathrm{Key}:=$ no inhibition; $+=$ mild inhibition $(0.5 \%-34 \%$ inhibition $) ;++=$ moderate inhibition (35\% - 54\% inhibition); +++ = good inhibition (55\% - 74\% inhibition); ++++ = high inhibition (75\% - 99\% inhibition); +++++ = complete inhibition; NA = not applicable; $\mathrm{a}=$ complete inhibition; $\mathrm{b}=$ high inhibition; $\mathrm{c}=$ good inhibition; $\mathrm{d}=$ moderate inhibition; $\mathrm{e}=$ mild inhibition; $\mathrm{f}=$ no inhibition.

Aspergillus niger, Aspergilus flavus and Fusarium oxysporum, respectively. Incorporation of $6 \mathrm{~mL}$ of extract recorded good inhibition (c) of Rhizopus stolonifer, it showed moderate inhibition (d) of Fusarium oxysporum and Penicillium marneffei, and mild inhibition (e) of Aspergillus niger and Aspergilus flavus, respectively. Addition of $7 \mathrm{~mL}$ recorded good inhibition of Rhizopus stolonifer, Fusarium oxysporum and Penicillium marneffei, it showed moderate inhibition of Aspergillus niger and Aspergilus flavus, respectively. Incorporation of $8 \mathrm{~mL}$ of extract recorded high inhibition (b) of Aspergillus niger and good inhibition (c) of Rhizopus stolonifer, Aspergilus flavus Fusarium oxysporum and Penicillium 
marneffei, respectively. Addition of $9 \mathrm{~mL}$ of extract recorded high inhibition (b) of the five fungi isolates, respectively. Incorporation of $10 \mathrm{~mL}$ extract recorded complete inhibition (a) of Fusarium oxysporum and high inhibition (b) of Rhizopus stolonifer, Aspergillus niger, Aspergilus flavus and Penicillium marneffei, respectively.

\subsubsection{Ethanol and Methanol solvents, Bench Extracted Synergistic Ten-Plants Inhibition Test on Isolates}

Table 6 and Table 7 present results of ethanol and methanol bench extracted synergistic ten plants inhibition test on isolates, the results are same with those obtained from cold aqueous synergistic extract. Addition of $1.0 \mathrm{~mL}$ of extracts in the culture media was able to inhibit Pseudomonas aeruginosa and Serratia marcescens mildly (e); Erwinia carotovora and Klebsiella oxytoca were inhibited moderately (d), respectively; the fungi isolates however showed no inhibition. Incorporation of $2.0 \mathrm{~mL}$ of the extract recorded complete inhibibition (a) of all the four bacteria isolates (Erwinia carotovora, Pseudomonas aeruginosa, Serratia marcescens and Klebsiella oxytoca); the fungi isolates were still not inhibited. Addition of $3.0 \mathrm{~mL}$ of extract showed mild inhibition (e) of Aspergillus niger and Penicillium marneffei without affecting the other three fungi; $4.0 \mathrm{~mL}$ incorporation of the extract showed mild inhibition (e) of Rhizopus stolonifer and Fusarium oxytoca; moderate inhibition (d) of Aspergillius niger, Aspergillus flavus and Penicillium marneffei. There was marked increase in the inhibition with the addition of $5.0 \mathrm{~mL}$ of the extract, showing good inhibition (c) for Rhizopus stolonifer, Aspergillus niger, Aspergilus flavus and Fusarium oxysporum; and high inhibition (b) for Penicilium marneffei. Incorporation of $6 \mathrm{~mL}$ of the extract showed good inhibition of Rhizopus stolonifer, Aspergillus niger and Fusarium oxysporum; and high inhibition of Aspergillus flavus and Penicillium marneffei, respectively. Addition of $7 \mathrm{~mL}$ and $8 \mathrm{~mL}$ of extract to test media showed good inhibition (c) of Rhizopus stolonifer and high inhibition (b) of Aspergillius niger, Aspergillus flavus, Fusarium oxysporum and Penicillium marneffei, respectively.

Incorporation of $9 \mathrm{~mL}$ recorded complete inhibition (a) of Fusarium oxysporum and Penicillium marneffei and high inhibition (b) of Rhizopus stolonifer, Aspergillus niger and Aspergilus flavus, respectively. Incorporation of $10 \mathrm{~mL}$ of extract recorded complete inhibition (a) of Rhizopus stolonifer, Fusarium oxysporum and Penicillium marneffei and high inhibition (b) of Aspergillus niger and Aspergilus flavus, respectively.

\subsubsection{Cold Aqueous Synergistic Ten-Plant Extract Inhibition Test on Weak Spores}

Table 8 shows result of cold aqueous synergistic ten plants extract inhibition test on weak spores; the two fungi, Aspergillus niger and Aspergillus flavus were not completely inhibited in the first treatment with the extract dose $(10 \mathrm{~mL})$ that completely inhibited the other three fungi (Rhizopus stolonifer, Fusarium oxysporum and Penicillium marneffei). The second and third treatments showed 
Table 6. Ethanol bench extracted synergistic ten plants inhibition test on isolates.

\begin{tabular}{|c|c|c|c|c|c|c|c|c|c|c|}
\hline \multirow{2}{*}{ Microorganism } & \multicolumn{10}{|c|}{ Extract volume applied } \\
\hline & $1 \mathrm{~mL}$ & $2 \mathrm{~mL}$ & $3 \mathrm{~mL}$ & $4 \mathrm{~mL}$ & $5 \mathrm{~mL}$ & $6 \mathrm{~mL}$ & $7 \mathrm{~mL}$ & $8 \mathrm{~mL}$ & $9 \mathrm{~mL}$ & $10 \mathrm{~mL}$ \\
\hline Rhizopus stolonifer & $--^{f}$ & $--^{\mathrm{f}}$ & $-^{\mathrm{f}}$ & $+^{e}$ & $+++^{c}$ & $+++^{c}$ & $+++^{c}$ & $++++^{\mathrm{b}}$ & $++++^{\mathrm{b}}$ & $+++++^{\mathrm{a}}$ \\
\hline Aspergillus niger & $-{ }^{f}$ & $--^{\mathrm{f}}$ & $+^{\mathrm{e}}$ & $++^{\mathrm{d}}$ & $+++^{c}$ & $+++^{c}$ & $++++^{\mathrm{b}}$ & $++++^{\mathrm{b}}$ & $++++^{\mathrm{b}}$ & $++++^{\mathrm{b}}$ \\
\hline Aspergillus flavus & $-^{\mathrm{f}}$ & $-\mathrm{f}$ & $-^{\mathrm{f}}$ & & $+++^{c}$ & $++++^{\mathrm{b}}$ & $++++^{\mathrm{b}}$ & $++++^{\mathrm{b}}$ & $++++^{\mathrm{b}}$ & $++++^{\mathrm{b}}$ \\
\hline Fusarium oxysporum & $--^{f}$ & $-^{\mathrm{f}}$ & $-^{\mathrm{f}}$ & & $+++^{c}$ & $+++^{c}$ & $++++^{\mathrm{b}}$ & $++++^{\mathrm{b}}$ & $+++++^{\mathrm{a}}$ & $+++++^{\mathrm{a}}$ \\
\hline Penicillium marneffei & $-{ }^{\mathrm{f}}$ & $-{ }^{f}$ & $+^{e}$ & $++^{\mathrm{d}}$ & $++++^{\mathrm{b}}$ & $++++^{\mathrm{b}}$ & $++++^{\mathrm{b}}$ & $++++^{\mathrm{b}}$ & $+++++^{\mathrm{a}}$ & $+++++^{\mathrm{a}}$ \\
\hline Erwinia carotovora & $++^{\mathrm{d}}$ & $+++++^{a}$ & $N A$ & $+^{e}$ & $N A$ & $N A$ & $N A$ & $N A$ & $N A$ & $N A$ \\
\hline $\begin{array}{c}\text { Pseudomonas } \\
\text { aeruginosa }\end{array}$ & $++^{\mathrm{d}}$ & $+++++^{\mathrm{a}}$ & $N A$ & $N A$ & $N A$ & $N A$ & $N A$ & $N A$ & $N A$ & $N A$ \\
\hline Serratia marcescens & $++^{\mathrm{d}}$ & $+++++^{\mathrm{a}}$ & $N A$ & $N A$ & $N A$ & $N A$ & $N A$ & $N A$ & $N A$ & $N A$ \\
\hline Klebsiella oxytoca & $++^{\mathrm{d}}$ & $+++++^{\mathrm{a}}$ & $N A$ & $N A$ & $N A$ & $N A$ & $N A$ & $N A$ & $N A$ & $N A$ \\
\hline
\end{tabular}

Summary of inhibition: $\mathrm{a}=9, \mathrm{~b}=15, \mathrm{c}=9, \mathrm{~d}=5, \mathrm{e}=6, \mathrm{f}=13 . \mathrm{Key}:=$ no inhibition; $+=$ mild inhibition $(0.5 \%-34 \%$ inhibition $)$; $++=$ moderate inhibition (35\% - 54\% inhibition); +++ = good inhibition (55\% - 74\% inhibition; ++++ = high inhibition (75\% - 99\% inhibition); +++++ = complete inhibition; NA = not applicable; $\mathrm{a}=$ complete inhibition; $\mathrm{b}=$ high inhibition; $\mathrm{c}=$ good inhibition; $\mathrm{d}=$ moderate inhibition; $\mathrm{e}=$ mild inhibition; $\mathrm{f}=$ no inhibition.

Table 7. Methanol bench extracted synergistic ten plants inhibition test on isolates.

\begin{tabular}{|c|c|c|c|c|c|c|c|c|c|c|}
\hline \multirow{2}{*}{ Microorganism } & \multicolumn{10}{|c|}{ Extract volume applied } \\
\hline & $1 \mathrm{~mL}$ & $2 \mathrm{~mL}$ & $3 \mathrm{~mL}$ & $4 \mathrm{~mL}$ & $5 \mathrm{~mL}$ & $6 \mathrm{~mL}$ & $7 \mathrm{~mL}$ & $8 \mathrm{~mL}$ & $9 \mathrm{~mL}$ & $10 \mathrm{~mL}$ \\
\hline Rhizopus stolonifer & $-{ }^{\mathrm{f}}$ & $-{ }^{\mathrm{f}}$ & $-{ }^{\mathrm{f}}$ & $+^{\mathrm{e}}$ & $+++^{\mathrm{c}}$ & $+++^{\mathrm{c}}$ & $+++^{c}$ & $++++^{\mathrm{b}}$ & $++++^{\mathrm{b}}$ & $+++++^{\mathrm{a}}$ \\
\hline Aspergillus niger & $-{ }^{\mathrm{f}}$ & $-{ }^{\mathrm{f}}$ & $+^{\mathrm{e}}$ & $++^{\mathrm{d}}$ & $+++^{\mathrm{c}}$ & $+++^{\mathrm{c}}$ & $++++^{\mathrm{b}}$ & $++++^{\mathrm{b}}$ & $++++^{\mathrm{b}}$ & $++++^{\mathrm{b}}$ \\
\hline Aspergillus flavus & $-{ }^{\mathrm{f}}$ & $-{ }^{\mathrm{f}}$ & $-{ }^{\mathrm{f}}$ & $++^{\mathrm{d}}$ & $+++^{\mathrm{c}}$ & $++++^{\mathrm{b}}$ & $++++^{\mathrm{b}}$ & $++++^{\mathrm{b}}$ & $++++^{\mathrm{b}}$ & $++++^{\mathrm{b}}$ \\
\hline Fusarium oxysporum & $--^{\mathrm{f}}$ & $--^{\mathrm{f}}$ & $--^{\mathrm{f}}$ & $+^{\mathrm{e}}$ & $+++^{\mathrm{c}}$ & $+++^{c}$ & $++++^{\mathrm{b}}$ & $++++^{\mathrm{b}}$ & $+++++^{\mathrm{a}}$ & $+++++^{\mathrm{a}}$ \\
\hline Penicillium marneffei & $--^{\mathrm{f}}$ & $--^{f}$ & $+^{\mathrm{e}}$ & $++^{\mathrm{d}}$ & $++++^{\mathrm{b}}$ & $++++^{\mathrm{b}}$ & $++++^{\mathrm{b}}$ & $++++^{b}$ & $+++++^{\mathrm{a}}$ & $+++++^{\mathrm{a}}$ \\
\hline Erwinia carotovora & $++^{\mathrm{d}}$ & $+++++^{\mathrm{a}}$ & $N A$ & $N A$ & $N A$ & $N A$ & $N A$ & $N A$ & $N A$ & $N A$ \\
\hline Pseudomonas aeruginosa & $++^{\mathrm{d}}$ & $+++++^{\mathrm{a}}$ & $N A$ & $N A$ & $N A$ & $N A$ & $N A$ & $N A$ & $N A$ & $N A$ \\
\hline Serratia marcescens & $++^{\mathrm{d}}$ & $+++++^{\mathrm{a}}$ & $N A$ & $N A$ & $N A$ & $N A$ & $N A$ & $N A$ & $N A$ & $N A$ \\
\hline Klebsiella oxytoca & $++^{\mathrm{d}}$ & $+++++^{\mathrm{a}}$ & $N A$ & $N A$ & $N A$ & $N A$ & $N A$ & $N A$ & $N A$ & $N A$ \\
\hline
\end{tabular}

Summary of inhibition: $\mathrm{a}=9, \mathrm{~b}=15, \mathrm{c}=9, \mathrm{~d}=5, \mathrm{e}=6, \mathrm{f}=13$. Key: $=$ no inhibition; $+=$ mild inhibition $(0.5 \%-34 \%$ inhibition $)$; $++=$ moderate inhibition (35\% - 54\% inhibition); +++ = good inhibition (55\% - 74\% inhibition); ++++ = high inhibition (75\% - 99\% inhibition); +++++ = complete inhibition; NA = not applicable; $\mathrm{a}=$ complete inhibition; $\mathrm{b}=$ high inhibition; $\mathrm{c}=\operatorname{good}$ inhibition; $\mathrm{d}=$ moderate inhibition; $\mathrm{e}=\mathrm{mild}$ inhibition; $\mathrm{f}=\mathrm{no}$ inhibition.

Table 8. Cold aqueous synergistic ten plants extract inhibition test on weak spores.

\begin{tabular}{lcccc} 
& \multicolumn{3}{c}{ Application times } \\
\cline { 2 - 4 } Microorganism & $1^{\text {st }}$ & $2^{\text {nd }}$ & $3^{\text {rd }}$ & $4^{\text {th }}$ \\
Aspergillus niger & + & + & + & - \\
Aspergillus flavus & + & + & + & -
\end{tabular}

Key: - = absence of growth; + = presence of growth. 
markedly reduced growth and retarded sporangium formation; seeding of the weak spores from third treatment plate onto a fresh and forth treatment plate yielded complete inhibition (no growth). This implies that to successfully keep out the fungi, treatment has to be repeated.

\subsection{Discussion}

Single plant extraction inhibition test (antimicrobial sensitivity test) results (Table 1) showed antifungal and antibacterial activity of the fresh plant leaves extracts in varying degrees. Single plant extract inhibited completely the growth of three organisms by two plants: Terminalia catapa at $100 \%$ and $10^{-1}$ showed complete inhibition (a) of Erwinia carotovora and Passiflora edulis at undiluted (100\%) concentration completely inhibited Rhizopus stolonifer and Penicillium marneffei. The result is in agreement with reports of several researchers who observed that, of the several methods adopted for controlling losses due to post harvest disease of yam; biological control method has been preferred in some cases because it is selective with no side effect and cheap. Resistance to biological control is rare and biological control agents are self-propagations and self-perpetuating [6] [35]; the advantage of plant extract products includes its local availability, presence of little or no toxicity to humans and simple preparation procedures [8] [28].

Cold aqueous synergistic ten plants extract inhibition Test on Isolates as shown in Table 2 recorded better inhibition. There was complete inhibition (a) of all the four bacteria isolates at $2 \mathrm{~mL}$ extract incorporation; $10 \mathrm{~mL}$ extract incorporation in media recorded complete inhibition (a) of three out of the five fungi isolates (Rhizopus stolonifer, Fusarium oxysporum and Penicillium marneffei and high inhibition (b) of the other two (Aspergillus niger and Aspergilus flavus), respectively. Further extract quantity addition was no longer necessary because at $10 \mathrm{~mL}$ incorporation the media was too soft, again in treatment, there is room for repeated dosage. The adoption of synergistic plant extract has been reported by other researchers as more effective, [36] [37].

Hot Aqueous Synergistic Ten Plants Extract Inhibition Test as shown in Table 3 , recorded reduced activity when compared to that of cold aqueous synergistic extract. This is most probably that some of the active ingredients are denatured by heat. This result is in agreement with the findings from other researchers, who observed that Zones of inhibition were also diminished by heating the extract, although its antimicrobial activity was not totally lost when heated [38] [39] [40].

Ethanol and Methanol Solvents Soxhlet extracted synergistic ten plants Inhibition Test on the Isolates as shown in Table 4 and Table 5 recorded reduced activity when compared to the hot aqueous extract (boiled extract); Heat employed extractions generally recorded less inhibition (Plates 1-3). This finding is in agreement with [39] who observed that all the influential factors (temperature, solvents, agitation speed, among others) might have the ability to enhance 


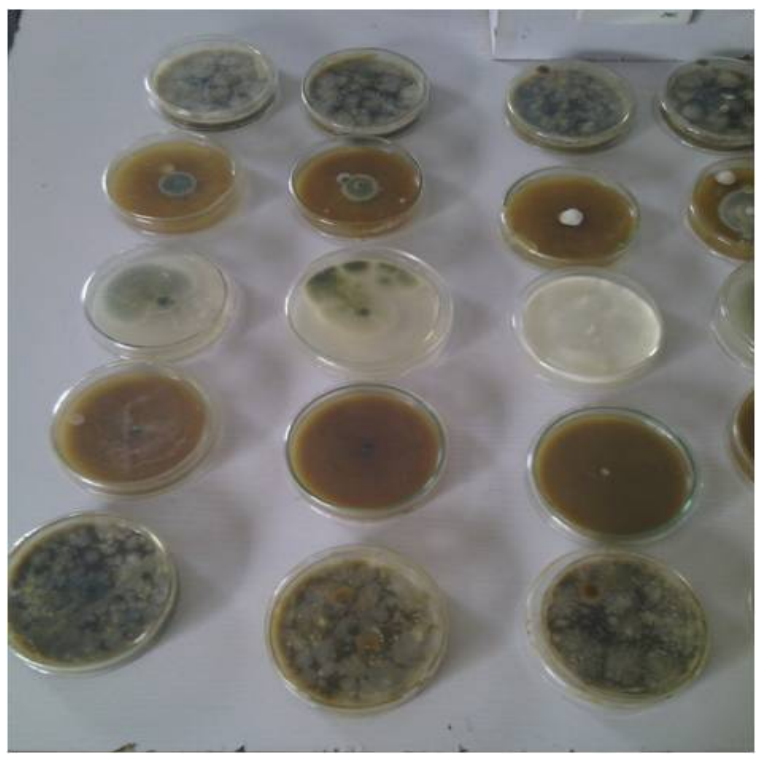

Plate 1. From Bottom to Top; Row 1: Non-inhibition by Soxhlet Methanol extracts on Fungi isolates (from right to left; Fusarium oxysporum, Aspergillus flavus and Penicillium marneffei); Row 2: Inhibition by Cold Aqueous Synergistic Ten-Plant extracts on Fungi isolates (from right to left; Fusarium oxysporum, Aspergillus flavus and Penicillium marneffei); Row 3: Fungi Control inoculations (from right to left; Fusarium oxysporum, Aspergillus flavus and Penicillium marneffei); Row 4: Inhibition by Boiled Synergistic Ten-Plant extracts on Fungi isolates (from right to left); Fusarium oxysporum, Aspergillus flavus and Penicillium marneffei. Row 5: Non-inhibition of Ethanol Soxhlet extracts on fungi isolates (from right to left; Fusarium oxysporum, Aspergillus flavus and Penicillium marneffei).

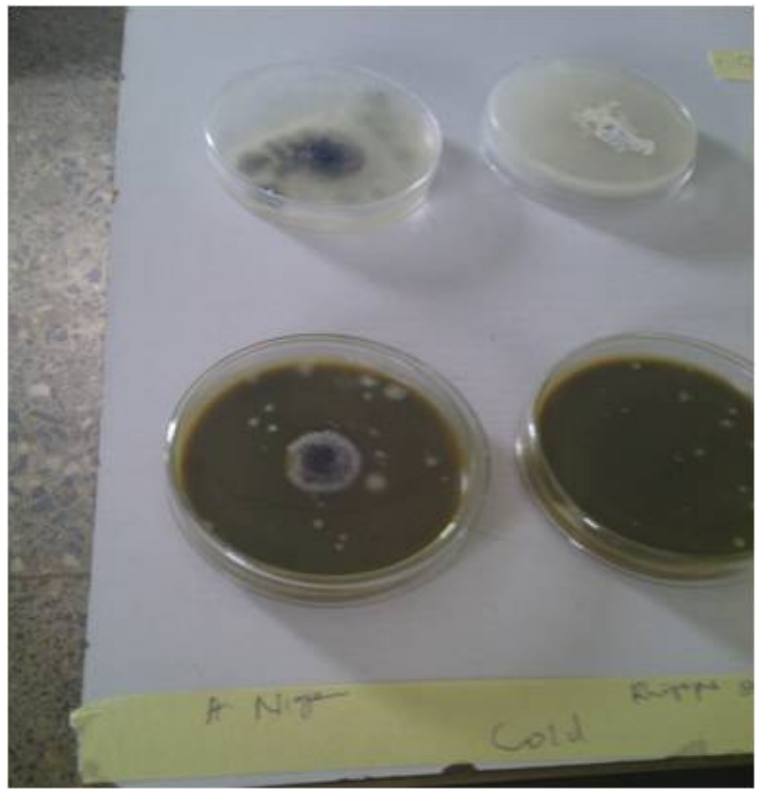

Plate 2. (from Left to right) Partial Inhibition of $A$. niger and Complete Inhibition of $R$. stolonifer by Synergistic Ten-Plants extract. 


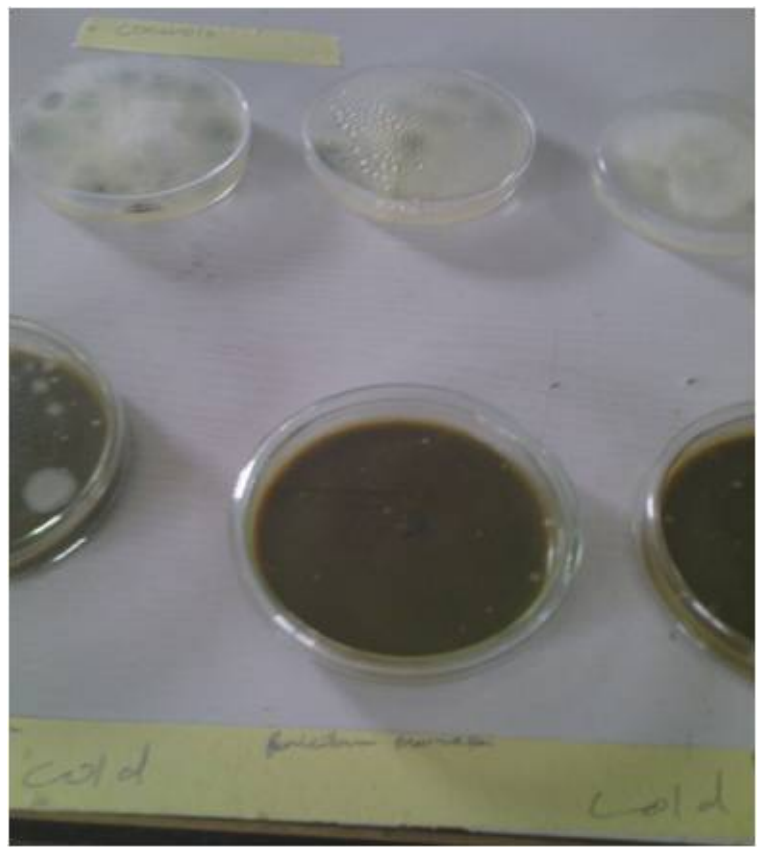

Plate 3. Complete inhibition of P. marneffei by Synergistic Ten-Plants extract.

extraction, but without proper judgment may cause degradation of compounds; also the Soxhlet extraction comes with disadvantage such as exposure to hazardous and flammable liquid organic solvents, with potential toxic emissions during extraction. Again solvents used in the extraction system need to be of high-purity, that might add to the extraction cost [40]; also they might not be environmental friendly and may contribute to pollution problem [1].

Room temperature Ethanol and methanol solvents extracted synergistic ten plants inhibition test on isolates as shown in Table 6 and Table 7 recorded inhibition activity that was same with that obtained with cold aqueous synergistic ten plants extract. This finding is in agreement with, [39] [40], who observed and recommended that, the use of water as extraction solvent should be preferred over organic solvents when application on food for preservation is the target.

Cold aqueous synergistic ten plants extract inhibition test on weak spores and subsequently derived weak spores reduced their resistance until they were completely inhibited after the third treatment as shown in Table 8. This agrees with [41] who observed that, bacterial are more susceptible to plant extracts than fungi. Also it is common knowledge that, most often treatment of fungi infections require repeated dosage and application of antifungal agent.

\section{Conclusion}

Single plant extract recorded complete inhibition of bacteria Erwinia carotovora by Terminalia catapa and fungi Penicillium marneffei by Passiflora edulis only; Pseudomonas aeruginosa and Klebsiella oxytoca recorded good inhibition (b) 
with Jatropha tanjorensis plant extract. Synergistic extract appears to enhance the complete inhibition of bacteria more than the fungi. The synergistic mixture comprising of all plants earlier used in single plant extractions gave better inhibition activity on the isolates. It was able to inhibit completely all the four bacteria isolates at $2 \mathrm{~mL}$ extract addition in culture media. All the fungi studied showed lower inhibition with aqueous synergistic ten plants extracts at lower concentrations when compared with aqueous single plant extracts and showed a gradual increase in inhibition with increase in concentration of the synergistic extract. Hot aqueous synergistic plants extract recorded poor inhibition of the isolates as compared to the cold. Soxhlet solvent extracted synergistic plants extract, however, recorded lower inhibition as compared to hot aqueous synergistic plants extract and cold aqueous synergistic ten plants extracts, respectively. Room temperature solvent extracted synergistic plants extracts recorded inhibition that was same as that obtained with cold aqueous synergistic plants extract.

\section{Acknowledgements}

We are grateful to the Management of Benue State University Makurdi and her Centre for Food Technology and Research, for the financial support and the facilities availed us for the successful conduct of this research. We appreciate the staff of Chemistry and Biological Sciences Departments for their assistance.

\section{Conflicts of Interest}

The authors declare no conflicts of interest regarding the publication of this paper.

\section{References}

[1] Anjum, M.A., Ahmed, N., Babita, C.H. and Gupta, P. (2016) Plant Extracts in Post-Harvest Disease Management of Fruits and Vegetables-A Review. Journal of Food Processing and Technology, 7, 592. https://doi.org/10.4172/2157-7110.1000592

[2] FAO (2008) Food and Agricultural Organisation Corporate Statistical Database.

[3] Adeniji, M.O. (1970) Fungi Associated with Storage Decay of Yam in Nigeria. Phytopathology, 60, 590-592. https://doi.org/10.1094/Phyto-60-590

[4] Arene, O.B., Nwakiti, A.O. and Okafor, N. (1985) The Chemical Basis of the Pathology of Yam Tuber. In: Osuji, G., Ed., Advances in Yam Research, Biochemical Society of Nigeria, 362.

[5] Okigbo, R.N. (2005) Biological Control of Post Harvest Fungal Rot of Yam (Dioscorea spp.) with Bacillus subtilis. Mycopathologia, 159, 307-314. https://doi.org/10.1007/s11046-004-2454-8

[6] Amusa, N.A., Adegbite, A.A., Muhammed, S. and Baiyewu, R.A. (2003) Yam Diseases and Its Management in Nigeria. African Journal of Biotechnology, 2, 497-502. https://doi.org/10.5897/AJB2003.000-1099

[7] Ijato, J.Y. (2011) Evaluation of Antifungal Effects of Extracts of Allium sativum and Nicotiana tobacum against Soft Rot of Yam (Dioscorea alata). Researcher, 3, 1-5. http://www.sciencepub.net/researcher/research0302/01_3730research0302_1_5.pdf 
[8] Cowan, M.M. (1999) Plant Products as Antimicrobial Agents. Clinical Microbiology Reviews, 12, 564-582. https://doi.org/10.1128/CMR.12.4.564

[9] Borris, R.P. (1996) Natural Products Research: Perspectives from a Major Pharmaceutical Company. Journal of Ethnopharmacology, 51, 29-38. https://doi.org/10.1016/0378-8741(95)01347-4

[10] Nascimento, G.G.F., Locatelli, J., Freitas, P.C. and Silva, G.L. (2000) Antibacterial Activity of Plant Extracts and Phytochemicals on Antibiotic-Resistant Bacteria. Brazilian Journal of Microbiology, 31, 247-256. https://doi.org/10.1590/S1517-83822000000400003

[11] Stockwell, C. (1988) Nature's Pharmacy. Century Hutchinson Ltd., London.

[12] Eisenberg, D.M., Kessler, R.C., Foster, C., Norlock, F.E., Calkins, D.R. and Delbanco, T.L. (1993) Unconventional Medicine in the United States: Prevalence, Costs and Patterns of Use. The New England Journal of Medicine, 328, 246-252. https://doi.org/10.1056/NEJM199301283280406

[13] Anesini, E. and Perez, C. (1993) Screening of Plants Used in Argentine Folk Medicine for Antimicrobial Activity. Journal of Ethnopharmacology, 39, 119-128. https://doi.org/10.1016/0378-8741(93)90027-3

[14] Martinez, M.J., Vasquez, S.M., Espinosa-Perez, C., Dias, M. and Herrera-Sanchez, M. (1994) Antimicrobial Properties of Argentatine A Isolated from Parthenium argentatum. Fitoterapia, 65, 371-372.

[15] Martinez, M.J., Betancourt, J., Alonso-Gonzalez, N. and Jauregui, A. (1996) Screening of Some Cuban Medicinal Plants for Antimicrobial Activity. Journal of Ethnopharmacology, 52, 171-174. https://doi.org/10.1016/0378-8741(96)01405-5

[16] Alonso-Paz, E., Cerdeiras, M.P., Fernandez, J., Ferreira, F., Moyna, P., Soubes, M., Vazquez, A., Veros, S. and Zunno, L. (1995) Screening of Uruguayan Medicinal Plants for Antimicrobial Activity. Journal of Ethnopharmacology, 45, 67-70. https://doi.org/10.1016/0378-8741(94)01192-3

[17] Devendra, B.N., Srinivas, N., Prasad Talluri, V.S.S. and Swarna, P.L. (2011) Antimicrobial Activity of Moringa oleifera Lam., Leaf Extract, against Selected Bacterial and Fungal Strains. International Journal of Pharma and Bio Sciences, 2, 13-18. http://www.ijpbs.net

[18] Abou-Karam, M. and Shier, W.T. (1990) A Simplified Plaque Reduction Assay for Antiviral Agents from Plants. Demonstration of Frequent Occurrence of Antiviral Activity in Higher Plants. Journal of Natural Products, 53, 340-344. https://doi.org/10.1021/np50068a011

[19] Afolayan, A.J. and Meyer, J.J.M. (1997) The Antimicrobial Activity of 3,5,7-Trihydroxyflavone Isolated from the Shoots of Helichrysum aureonitens. Journal of Ethnopharmacology, 57, 177-181. https://doi.org/10.1016/S0378-8741(97)00065-2

[20] Ahmad, A., Davies, J., Randall, S. and Skinner, G.R.B. (1996) Antiviral Properties of Extract of Opuntia streptacantha. Antiviral Research, 30, 75-85. https://doi.org/10.1016/0166-3542(95)00839-X

[21] Ahmed, A.A., Mahmoud, A.A., Williams, H.J., Scott, A.I., Reibenspies, J.H. and Mabry, T.J. (1993) New Sesquiterpene $\alpha$-methylene Lactones from the Egyptian Plant Jasonia candicans. Journal of Natural Products, 56, 1276-1280. https://doi.org/10.1021/np50098a011

[22] Amoros, M., Simoes, C.M.O. and Girre, L. (1992) Synergistic Effect of Flavones and Flavonols against Herpes Simplex Virus Type-1 in Cell Culture. Comparison with the Antiviral Activity of Propolis. Journal of Natural Products, 55, 1732-1740. 
https://doi.org/10.1021/np50090a003

[23] Anonymous (1998) Commission on Dietary Supplement Labels Issues Final Report. Journal of the American Dietetic Association, 98, 270. https://doi.org/10.1016/S0002-8223(98)00064-9

[24] Anonymous (1997) FANSA Releases Statement about Dietary Supplement Labeling. Journal of the American Dietetic Association, 97, 728-729.

[25] Anonymous (1997) First IND Submitted with FDA for an Herbal Pharmaceutical. AIDS Weekly Plus 18.

[26] Ogundana, S.K., Coxon, D.T., Denis, C. and Nagavic, S.H.Z. (1980) Natural Antifugal Compounds from the Peel of Yam Tubers. Proceedings of the 6th Symposium of the TRC International Potato Center, 616.

[27] Okigbo, R.N., Agbata, C.A. and Echezona, C.E. (2010) Effects of Leaf Extracts of Azadirachta indica and Chromolaena odorata on Post Harvest Spoilage of Yams in Storage. Current Research Journal of Biological Sciences, 2, 9-12.

[28] Okigbo, R.N. and Nmeka, I.A. (2005) Control of Yam Tuber with Leaf of Xylopia aethiopica and Zingiber officinale. African Journal of Biotechnology, 4, 804-807.

[29] Shiriki, D., Obochi, G.O., Eke, M.O. and Shambe, T. (2017) Postharvest Loss Control: Synergistic Plants Extract Inhibition of Ten Microbial Yam Rot Organisms. Food and Nutrition Sciences, 8, 725-732. https://doi.org/10.4236/fns.2017.87051

[30] Amadioha, A.C. and Obi, V.I. (1999) Control of Anthracnose Disease of Cowpea by Cymbopogon citrates and Ocimum gratissimum. Acta Phytopathologica et Entomologica Hungarica, 34, 85-89.

[31] Banso, A. and Sani, A. (2003) Antibacterial Effect of Leaf Extract of Ricinus communis. African Scientist, 4, 129-133.

[32] Delahaye, C., Rainford, L., Nicholson, A., Mitchell, S., Lindo, J. and Ahmad, M. (2009) Antibacterial and Antifungal Analysis of Crude Extract from the Leaves of Callistemon viminalis. J. Med. And Biol. Scs. 3, 1-7.

[33] Nagamani, J.E., Vidya, S.D. and Syeda, H.B. (2014) A Study on Antioxidant and Antimicrobial Properties of Bombax Ceiba pentandra Seed Extract. World Journal of Pharmacy and Pharmaceutical Sciences, 3, 692-706. http://www.wipps.com

[34] Ezeibekwe, I.O. and Ibe, A.E. (2010) Fungal Organisms Associated with Yam (Dioscorea rotundata, poir) Rot at Owerri, Imo State of Nigeria. Journal of Molecular Genetics, 2, 1-5. https://doi.org/10.3923/jmolgene.2010.1.5

[35] Okigbo, R.N. and Ikediugwu, F.E.O. (2000) Studies on Biological Control of Postharvest Rot of Yam (Diocorea spp.) with Trichoderma viride. Journal of Phytopathology, 148, 351-355. https://doi.org/10.1111/j.1439-0434.2000.tb04786.x

[36] Tijjani, A. (2013) Antibiotic Synergy as a New Strategy for Combacting Drug Resistant Bacteria.

[37] Wendakoon, C., Calderon, P. and Gagnon, D. (2012) Evaluation of Selected Medicinal Plants Extracted in Different Ethanol Concentrations for Antibacterial Activity against Human Pathogens. Journal of Medicinally Active Plants, 1, 60-68. http://scholarworks.umass.edu/jmap/vol1/iss2/4

[38] Akrayil, H.F.S. (2013) Antibacterial Potency of Aqueous Plant Extracts against Streptococcus Mutans. Medical Journal of Islamic World Academy of Sciences, 22, 85-89. https://doi.org/10.12816/0008177

[39] Irobi, O.N. (1997) Antibiotic Properties of Ethanol Extract of Chromolaena odorata (Asteriaceae). International Journal of Pharmacognosy, 35, 111-115. https://doi.org/10.1076/phbi.35.2.111.13287 
[40] Azwanida, N.N. (2015) A Review on the Extraction Methods Use in Medicinal Plants, Principle, Strength and Limitation. Medicinal and Aromatic Plants, 4, 3. https://doi.org/10.4172/2167-0412.1000196

[41] Czerwińska, E. and Szparaga, A. (2015) Antibacterial and Antifungal Activity of Plant Extracts. Annual Set the Environment Protection, 17, 209-229. 